\title{
Detecting individual gravity modes in the Sun: Chimera or reality?
}

\author{
Rafael A. García* \\ Laboratoire AIM, CEA/DSM - CNRS - Université Paris Diderot - IRFU/SAp, \\ 91191 Gif-sur-Yvette Cedex, France \\ email: rafael.garcia@cea.fr
}

\begin{abstract}
Over the past 15 years, our knowledge of the interior of the Sun has tremendously progressed by the use of helioseismic measurements. However, to go further in our understanding of the solar core, we need to measure gravity $(\mathrm{g})$ modes. Thanks to the high quality of the Doppler-velocity signal measured by GOLF/SoHO, it has been possible to unveil the signature of the asymptotic properties of the solar g modes, thus obtaining a hint of the rotation rate in the core (García et al. 2007, 2008a). However, the quest for the detection of individual $g$ modes is not yet over. In this work, we apply the latest theoretical developments to guide our research using GOLF velocity time series. In contrary to what was thought till now, we are maybe starting to identify individual low-frequency g modes...
\end{abstract}

Keywords. Sun: helioseismology, Sun: oscillations, Sun: interior, Sun: rotation

\section{Observations and analysis}

Gravity modes are very sensitive to the structure (e.g. Basu et al. 2009, García et al. 2008c) and the dynamics (e.g. Mathur et al. 2008) of the radiative zone and, in particular, to the inner core of the Sun. There have been many attempts to look for them without so far an undisputed detection of such modes (Appourchaux et al. 2009), although some interesting peaks and patterns have been detected with GOLF and VIRGO (e.g. TurckChièze et al. 2004, Jiménez \& García 2009) with a high confidence level.

A 4500-day GOLF time series (Gabriel et al. 1995) starting on April 11, 1996 and calibrated into velocity (García et al. 2005) has been used to compute a single, full resolution power spectrum density (PSD) in spite of the different sensitivity to the visible solar disk between the blue- and the red-wing GOLF measurements (see for further details García et al. 1998, Ulrich et al. 2000). To increase the signal-to-noise ratio, we have also smoothed the PSD with a $41-\mathrm{nHz}$ boxcar function as it is commonly done in asteroseismology when the signal is weak (e.g. Michel et al. 2008).

\section{Discussion}

Several of the highest peaks between 60 and $140 \mu \mathrm{Hz}$ are located around the theoretical frequencies of the dipole modes obtained by the Saclay seismic model (e.g. Mathur et al. 2007). Moreover, varying the splitting of the modes from 1 to 5 times the rotation rate of the radiative region, $\Omega_{\mathrm{rad}}$, we notice that there is a quasi complete sequence of peaks matching the model when the splitting of these modes is around $4.5 \Omega_{\mathrm{rad}}$ (see Fig.1). This could be the first time that individual g modes are identified in the Sun. For example, the candidate mode $\ell=1, \mathrm{n}=-4$ has an amplitude of $1.8 \pm 0.4 \mathrm{~mm} / \mathrm{s}$ (with a signal-to-noise ratio of $\sim 4$ ) which is close to the latest theoretical predictions (Belkacem et al. 2009). 


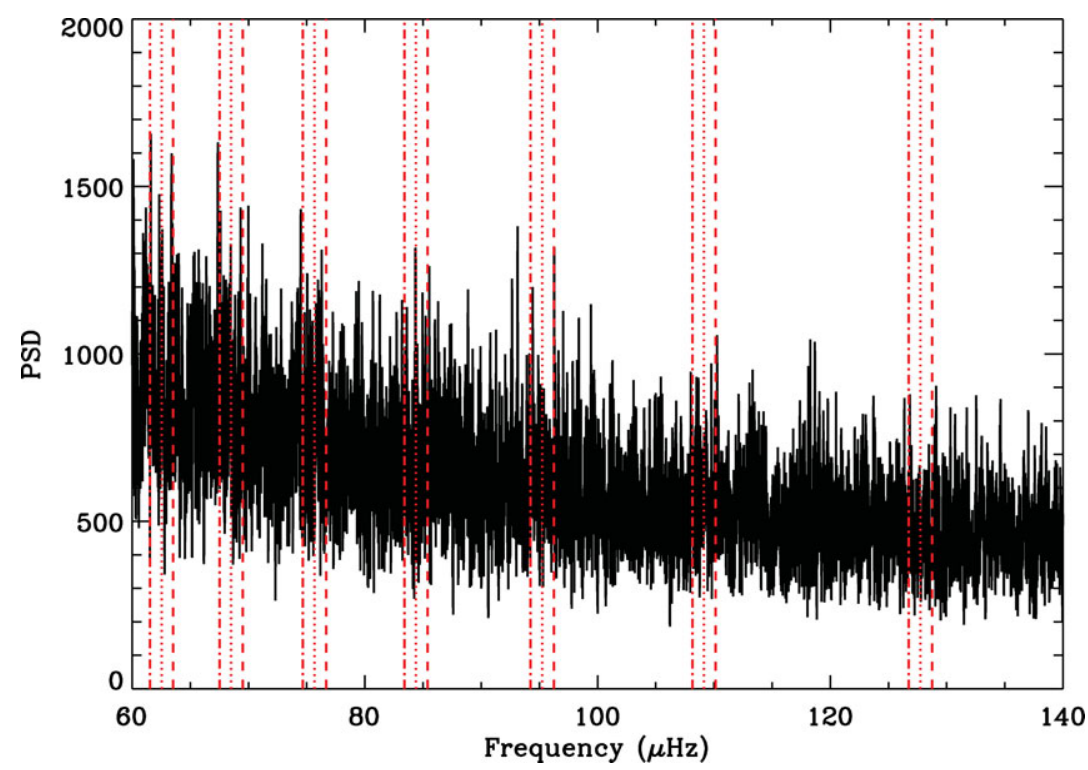

Figure 1. GOLF PSD. The dotted vertical lines are the central frequencies of the dipole $g$ modes computed by the Saclay seismic model. The vertical dashed and dot-dashed lines are the rotational split components with a rotational splitting 4.5 times larger than in the radiative zone above $0.2 R_{\odot}$.

The splittings can be estimated for each individual g-mode candidate. However, it is very difficult to obtain an accurate inference of the rotational profile of the core (García et al. 2008b, Mathur et al. 2009). The rotation rate in the core could be up to $\sim 7 \Omega_{\mathrm{rad}}$, since $\sim 60 \%$ of the rotational kernel of these g modes is located in the inner core.

\section{References}

Appourchaux, T., Belkacem, K., Broomhall, A.-M. et al. 2009, ARA\&A, arXiv:0910.0848

Basu, S., Chaplin, W. J., Elsworth, Y., New, R., \& Serenelli, A. M. 2009, ApJ, 699, 1403

Belkacem, K., Samadi, R., Goupil, M. J., Dupret, M. A. 2009, A\&A 494, 191

Gabriel, A. H., Grec, G., Charra, J. et al. 1995, Sol. Phys. 162, 61

García, R. A., Roca Cortés, T., \& Régulo, C. 1998, A\&GAS 128, 389

García, R. A., Turck-Chièze, S., Boumier, P. et al. 2005, A\& A 442, 385

García, R. A., Turck-Chièze, S., Jiménez-Reyes, S. J. et al. 2007, Science 316, 1591

García, R. A., Jiménez, A., Mathur, S. et al. 2008a, Astron. Nachrichten 329, 476

García, R. A., Mathur, S., Ballot, J. et al. 2008b, Sol. Phys. 251, 119

García, R. A., Mathur, S., \& Ballot, J. 2008c, Sol. Phys 251, 135

Jiménez, A. \& García, R. A. 2009, ApJS 184, 288

Mathur, S., Turck-Chièze, S., Couvidat, S., García, R. A. 2007, ApJ 668, 594

Mathur, S., Eff-Darwich, A., García, R. A.x, Turck-Chièze, S. 2008, A\&SA 484, 517

Mathur, S., García, R. A., \& Eff-Darwich, A. 2009, ASSP, arXiv:0902.4142

Michel, E., Baglin, A., Auvergne, M. 2008, Science 322, 558

Turck-Chièze, S., García, R. A., Couvidat, S. et al. 2004, A\& A 484, 517

Ulrich, R. K., García, R. A., Robillot, J.-M., et al. 2000, A\&̈A 364, 799

*In collaboration with: J. Ballot, A. Eff-Darwich, R. Garrido, A. Jiménez, S. Mathis, S. Mathur, A. Moya, P. L. Pallé, C. Régulo, D. Salabert, K. Sato, J. C. Suárez and S. Turck-Chièze 